\title{
Axiomatic Design for an Efficient Development of Optimized RPM Systems
}

\author{
Beniamino Pacifici, Chiara Parretti*, Andrea Girgenti, and Paolo Citti \\ Guglielmo Marconi University, Department of Innovation and Information Engineering, via Plinio 44, Rome, Italy
}

\begin{abstract}
New communication technologies and development of increasingly advanced systems for remote assistance create important opportunities related to the spread of e-health systems and devices for health monitoring and healthcare integration. Despite the increasingly widespread use of Remote Patient Monitoring (RPM) systems, especially in presence of chronic diseases, users' needs and requests are often disregarded due to poor customization of solutions. This is mainly due to the lack of truly interoperable tools allowing for horizontal integration between the various medical and specialized instruments. The proposed model works as a useful approach to ensure that the development of new solutions shall be partially guaranteed. The Axiomatic Design method is a suitable tool to identify the best solutions to meet users' needs.
\end{abstract}

\section{Introduction}

Long-term sustainability of health care provision raises serious concerns, due to increase in chronic diseases and gradual aging of population, which is making the provision of healthcare assistance more and more burdensome. To cope with this challenge, attention to healthcare management and efficiency of systems for therapies management have become a primary objective.

Developing a network, which provides the integration of health and social services with interoperable devices/systems, works as an optimized resource management tool. This network contributes not only to make the healthcare more tailored to the patient, but also to develop a more efficient and widespread prevention system. Moreover, these services allow remote treatment of chronic diseases and offer tools to healthcare providers to make clinical decisions faster and more reliable [1].

The progress of Remote Patient Monitoring solutions (RPM) is strongly stimulated by the simultaneous presence of socio-cultural factors that drive the market towards this direction:

- Users are progressively more willing to use wireless health monitoring systems,

- Governments push towards an optimization of healthcare costs,

- Average lifespan of people with chronic diseases to be treated is increasing, due to gradual aging of population,

- There are monitoring systems and sensors on the market, with an increasing diagnostic capacity for a growing number of chronic diseases and issues.

This article addresses the development of RPM solutions, clustered depending on users' needs, by means of a holistic approach. In this case, the AD methodology is an excellent help, especially in presence of a complex system, to analyze and transform Customer Needs (CNs) into Functional Requirements (FRs) and Design Parameters (DPs). Specifically, an application of the axiom of information is hereby proposed, using the Function Point Technique [2-7]. This methodology is used to measure the information content of FRs and DPs mutual relationships. Therefore, in this complex environment, it is possible to develop the most robust solution among the many ones that are possible thanks to $\mathrm{AD}$ methodology; moreover, among all of them, $\mathrm{AD}$ allows to choose the one that better meets the needs expressed by the users.

\section{State of the art}

There is a wide range of services and applications that are currently used with mobile devices to provide remote health care services. These systems can be categorized into two macro areas based on the type of service they provide: user services and systems meant to optimize the national health service. The first group includes services and applications which are used directly by individual patients and people interested in monitoring their health. The second group includes solutions that do not interact directly with patients, yet are implemented to improve the efficiency of health care (i.e.: services developed to support healthcare and/or administrative staff) $[1,8]$.

As of today, the most developed part of the first group is the remote monitoring of health conditions [910]. In Europe and the United States only, more than 200 million people are suffering of pathologies for which remote monitoring can be an optimal solution from a clinical standpoint [11-12]. These already identified users can add up new types of users, for whom solutions have not yet been implemented, such as patients who are

* Corresponding author: c.parretti@unimarconi.it 
interested in fetal monitoring, healthy people groups who are interested in monitoring their health and people who are interested in preventive screening [11-12]. RPM systems have several advantages beyond healthcare costs reduction, which have been highlighted by several studies conducted on both patients and healthcare professionals:

- there is an increasingly patient satisfaction with health systems, as well as an improvement in the quality of life;

- there is an improvement in patients' better willingness to follow the treatments prescribed by the doctors;

- there is a simplification of work for healthcare staff;

- there is a reduction in emergency and hospitalization interventions.

Despite the development of many solutions, which offer several benefits, these are still sectoral and barely characterized for specific groups of users.

\section{Challenges}

The diffusion of RPM systems is slowed down by multiple factors that cause a lag in their large-scale adoption (Fig.1). The lack of interoperability and standards limits the possibility of integration between medical/specialist tools and those tools aimed at improving the quality of life of patients. ISO/IEEE 11073 Health Informatics-Medical/health device communication standards enable communication between medical, healthcare and wellness devices, as well as between external computer systems. However, these standards identify requirements related to small devices with limited resources of processor, memory and power [13, 14]. This leads to a slowdown in the development and spread of these services, compromising the quality of information and generating cost increases.

\begin{tabular}{|c|c|c|c|c|}
\hline & \multicolumn{4}{|c|}{ Challenges } \\
\hline Users & Regulatory & Economic & Structural & Technological \\
\hline $\begin{array}{l}\text { Health care } \\
\text { provider }\end{array}$ & \multirow{3}{*}{$\begin{array}{l}\begin{array}{l}\text { Lack of } \\
\text { clarity on } \\
\text { certification }\end{array} \\
\text { Lack of } \\
\text { Interoperability }\end{array}$} & $\begin{array}{l}\text { Need for further } \\
\text { evidence } \\
\text { Conflicting } \\
\text { incentives } \\
\text { Change } \\
\text { management }\end{array}$ & $\begin{array}{l}\text { Low cohesion } \\
\text { across levels } \\
\text { and regions }\end{array}$ & $\begin{array}{l}\text { Late involvement } \\
\text { of doctors in } \\
\text { solution design }\end{array}$ \\
\hline $\begin{array}{l}\text { Solutions } \\
\text { vendor }\end{array}$ & & & & Interoperability \\
\hline $\begin{array}{l}\text { Mobile } \\
\text { service } \\
\text { provider }\end{array}$ & & $\begin{array}{l}\text { Lack of clear } \\
\text { reimbursement } \\
\text { mechanism }\end{array}$ & & $\begin{array}{l}\text { Standardization } \\
\text { Interoperability }\end{array}$ \\
\hline $\begin{array}{l}\text { Medical } \\
\text { devices } \\
\text { vendor }\end{array}$ & \multirow{2}{*}{$\begin{array}{l}\text { Lack of } \\
\text { compensation } \\
\text { mechanism }\end{array}$} & & & $\begin{array}{l}\text { Standardization } \\
\text { Interoperability }\end{array}$ \\
\hline $\begin{array}{l}\text { Doctors/ } \\
\text { Patients }\end{array}$ & & $\begin{array}{l}\text { Lack of } \\
\text { awareness of } \\
\text { system benefits } \\
\text { for the Doctors } \\
\text { and patients }\end{array}$ & & $\begin{array}{l}\text { Significant } \\
\text { training needs }\end{array}$ \\
\hline
\end{tabular}

Fig. 1. Summary of the main barriers to the development of mobile health systems.

Moreover, there are no unified rules for mobile application management in health and welfare industry, while from a legal perspective there is no adequate guarantee for the full protection of recorded data [15]. Additionally, there are other types of challenges such as:
- Political barriers: national and local health systems have different standards, while health policies often do not encourage the homogenization of systems,

- Definition barriers: healthcare and medicine are complex areas from a variety of perspectives, so both categories are difficult to define and categorize due to cultural, social, and contextual differences. In addition, medicine is a rapidly evolving field, thus mobile health standards shall adapt rapidly to contextual evolution with frequent revisions,

- User barriers: healthcare providers are often unsatisfied of devices currently available on the market because they are too sectoral and are not useful for patients' management; also end users often know that systems currently proposed are too complex.

\section{Health systems functionality}

The analysis of global context, regarding both users' needs and health systems requirements, suggests that the creation of a network for health and social services and integrated devices/interoperable systems, should focus on the following types of functionality [16]:

- health monitoring and comfort, such as systems related to patient monitoring, through direct employment of health professionals, when the complex/chronic patients have left the hospital. These systems should create a continuous supervision of the user's situation by means of various indicators, recorded and analyzed. Thus, these devices can greatly enhance the users' perception of their security, since they receive ongoing monitoring of their situation;

- Safety and speed of interventions in emergencies [17]: the systems enable the monitoring of early warning indicators related to critical situations (i.e. usage of bathroom and no long-term movement), through which it is possible to recognize false alarms thus avoiding unnecessary trips to the hospital,

- Knowledge of useful information: qualified support systems at home will help users in the resolution of everyday problems and concerns or to receive instructions and assistance from family,

- Support the performance of an activity: organization of daily activities that allow to smartly manage: time, nutrition, hydration, taking medication, etc. based on user specificity;

- Simplification of necessary actions, that being systems for automatic reordering of drugs with verification of stocks present together with the eating plan,

- Prevention tips and stimulus: systems can suggest how to perform normal daily activities to get a rehabilitative effect or make the necessary fun activities (i.e.: using a video games approach often used in rehabilitation contexts [18] for operations to be carried out by children). Self-testing systems monitor the current situation and its evolution through time,

- Compensation and assistance: systems can address/alleviate difficulties due to a certain disease related to environmental conditions. Programs designed for highly complex patients (for example, those with a functional or multiple disability) provide the 
employment of installed devices that control their ability to live independently.

\section{Axiomatic Design for RPM systems development}

This article deals with the methodology of ObjectOriented Analysis and Design (OOAD) within the Axiomatic Design (AD) approach [19], applied to the design of a software that is able to find the best solution for customizing RPM systems starting from basic interoperable elements. This methodology simplifies the integration of $\mathrm{AD}$ approach into the software development through the Object-Oriented Programming (OOP) technique.

OOAD is a technical approach for analyzing and designing an application, system, or business by applying the object-oriented paradigm, as well as using visual modeling throughout the development life cycles to foster better stakeholder communication and product quality.

$\mathrm{AD}$ is a top-down design methodology (from general down to more detailed elements) starting from the Customer Needs (CNs), the Functional Requirements (FRs) can be defined after them, then the Design Parameters (DPs) as well. If the analysis is too abstract, a FR decomposition is performed and then corresponding DPs are identified. This process continues until the level of detail required for the project is reached $[2,20]$.

Axiomatic Design is based on the verification of axioms of independence and information. The axiom of independence guarantees design solutions in which the relationships between FRs and DPs are uncoupled or decoupled. This axiom states that when multiple FRs exist, the design solution should satisfy the condition with a diagonal design matrix. Thus, each DP will affect only its associated FR with no coupling occurring in the off-diagonal elements. This type of solution is said to be uncoupled. When independence is not achieved, there are two cases. In one case the design will be decoupled, meaning that the matrix can be rearranged to obtain a triangular state; in the other case, when off-diagonal elements exist and the matrix cannot be rearranged to a triangular state, the design is said to be coupled. An acceptable design is either uncoupled or decoupled [21].

The axiom of information allows to select the most robust solution, whenever there are multiple solutions, which corresponds to the project with the lowest information content. The concept is to find the system that satisfies FRs through DPs with the highest probability.

Thus, the designer may also select the solution, among the different functional substantial decompositions, requiring the least effort in terms of resources and development time. This approach is the same as choosing the functional decomposition with the lowest information content, meeting the same functional requirements at the same time [6].

However, in the software design field, Axiomatic Design is often applied through the verification of the independence axiom alone. Solutions identified this way are coherent from a design point of view but are not necessarily the most robust in a conceptual sense. Scientific literature on this subject often justifies the nonapplication of the information axiom, indicating difficulty of quantifying the content of software information [19]. In this article, an application of the axiom of information is proposed, using the Function Point Technique [2-7].

This methodology is useful to measure the information content of FRs and DPs relations. Therefore, in this complex system, the application of AD allows the development of the most robust solution among the many possible, and above all, the one that better meets the needs expressed by users.

A function point is a "unit of measurement" to express the amount of business functionality an information system (as a product) provides to users. Using a standardized set of basic criteria, each function is a numeric index according to its type and complexity. These indices are totaled to give an initial measure of size, which is then normalized by incorporating several factors relating to the software as a whole. The result is a single number called the Function Point index, which measures the size and complexity of the software product.

To sum up, the function point technique provides an objective, comparative measure that assists in the evaluation, planning, management and control of software production.

\section{Holistic framework for the development of RPM systems based on user needs}

The proposal of this article is the creation of a system able to align RPM systems and customers' needs (whether they are patients or healthcare professionals). The scheme, shown below in fig. 2, is a concept of the followed approach.

Customers need to be grouped in clusters to offer a different type of application related to same scope.

Requirements and characteristics of systems must be classified according to users' type (e.g. children, elderly, healthcare professionals, etc.). This is a key step during the procedure of customizing the service: having responses to demands expressed not only in terms of technology offered, but above all in usability of software or applications interface. This avoids reticence in system adoption and provides customer satisfaction.

In this phase, the clusters of customers can be identified with the aid of Lean Six Sigma (LSS) [22] instruments, based on the transformation from Voice Of Customer (VOC) to Functional Requirements and Design Parameters [23-28]. In this specific case, the Quality Function Deployment (QFD) tool helps transforming the users' needs into functional requirements, then subsequently applying the Axiomatic Design 


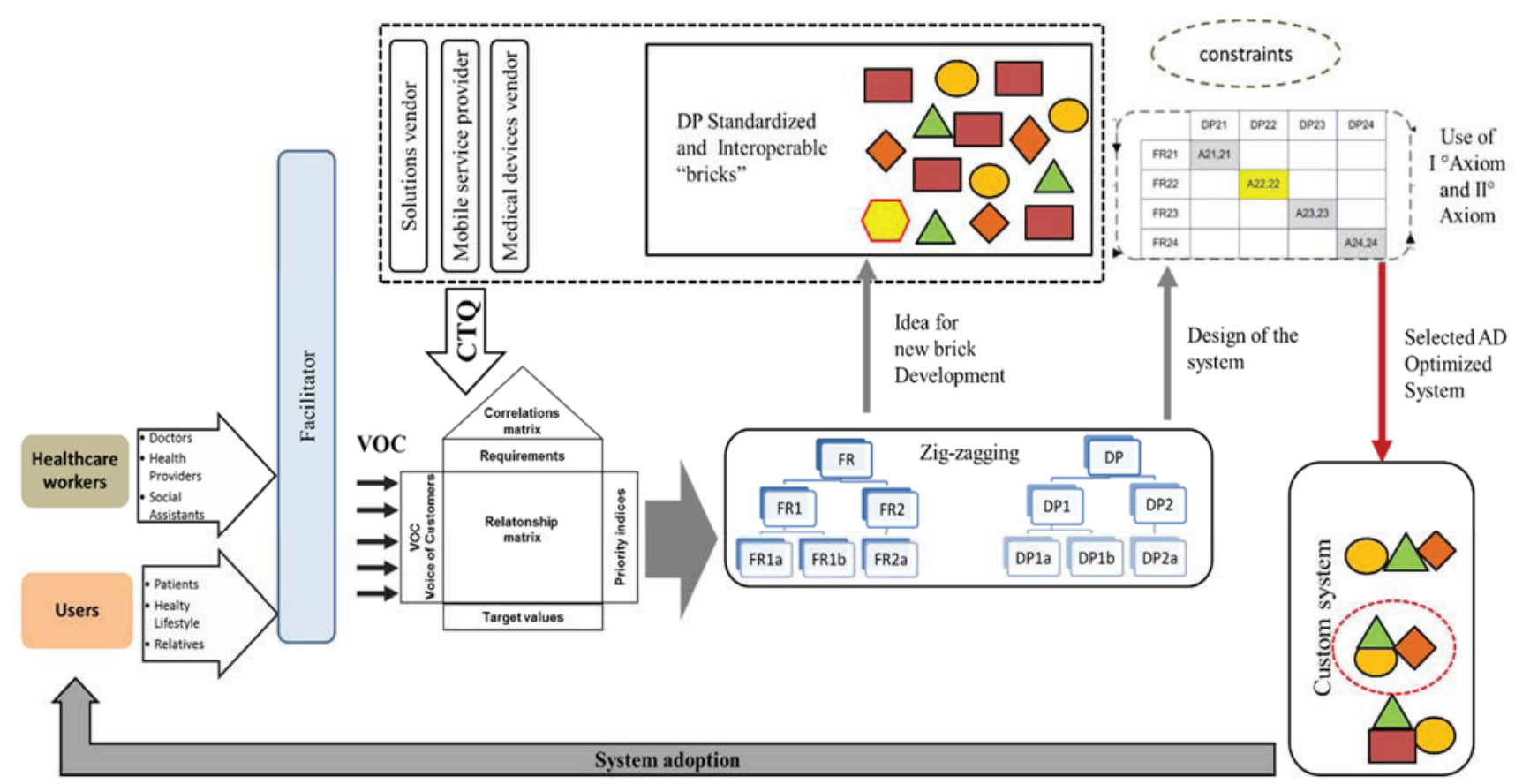

Fig. 2. Holistic Framework for the development of RPM systems based on user needs.

methodology. The effective versatility of $\mathrm{AD}$ has also been demonstrated by other specific applications not only in healthcare contexts but also in optimizing processes [29] and products [30].

According to the fig. 2, required FRs are also generated through continuous exchange of information that is collected by healthcare professionals. The involvement of healthcare professionals in this first phase reduces risks of inadequate construction of software features and avoids missing important information to be caught. Moreover, to make eHealth systems effective, coordination between various healthcare actors is crucial, starting from doctors among healthcare professionals, patients, informal caregivers until end users [31]. Health information provided by devices should be the one needed by healthcare operators to evaluate clinical situation, and must remain the same with no dependence from the user's cluster origin. For example, if a cardiovascular monitoring is required to monitor blood pressure, this value should be monitored whether it is an RPM system developed for the elderly users, or is developed for a pregnant woman.

All the users' and healthcare professionals' requirements are transformed through the aid of IT operators; using these specifications, they will develop elementary "bricks", which represent standardized and interoperable elements of RPM systems. The developers will assemble standardized bricks to organize their composition based on customer requests. In this phase, the QFD tool is also useful to identify the characteristics of the new bricks; in fact, it provides the weight of the technical performances needed by the specified cluster of users.

Now, the AD methodology is applied to ensure robustness of the RPM solution selected and assembled, more specifically by using methodology of ObjectOriented Programming applied to software design. The last phase of designing with $\mathrm{AD}$ is the identification of a decomposition between FRs and DPs, which corresponds to the conceptual process of system to be implemented in the software. Therefore, the output of AD design phase becomes the input for the software system programmers. Now, using OOP programming technique, which is a bottom-up system that starts from detail and goes to general, the best robust RPM solution can be obtained using the best single interoperable elements. The logic of system is the first phase of the software lifecycle.

To understand $\mathrm{AD}-\mathrm{OOAD}$, it is necessary to review the definitions of the words used in OOP and their equivalent words in Axiomatic Design. The fundamental construct for the object-oriented method is the "object", which is equivalent to FRs. Object-Oriented Programming decomposes a system into objects. Objects "encapsulate" both data (equivalent to DPs), and method (equivalent to relationship between FRi and DPi) in a single entity. Object retains certain information on how to perform certain operations, using the input provided by the data and the method embedded in the object. In terms of $\mathrm{AD}$, this is equivalent to say that an object is [32]:

$$
F R i=A i j \cdot D P j
$$

The concept is to design a RPM system that meets the FRs through DPs, by measuring the information contents of relations between FRs and DPs through the Function Point technique. At each level of decomposition of the FRs, an estimate can be made in terms of function points. In general, there is a certain correspondence between FRs, DPs and the constituent elements of the calculation in the function point. For instance, let us consider the functional decomposition represented by the correlation matrix between FRs and DPs:

*Corresponding author: c.parretti@unimarconi.it 


$$
\left\{\begin{array}{l}
F R 1 \\
F R 2 \\
F R 3
\end{array}\right\}=\left[\begin{array}{ccc}
A_{11} & 0 & 0 \\
0 & A_{22} & 0 \\
0 & 0 & A_{33}
\end{array}\right] \cdot\left\{\begin{array}{l}
D P 1 \\
D P 2 \\
D P 3
\end{array}\right\}
$$

To illustrate the correspondence, we must consider that each functional decomposition can be represented with a module-junction structure diagram. Each module of the diagram includes property details and functionalities associated with them [19, 20, 31]. Therefore, a generic module (M) is defined as the row of design matrix that yields the FR of the row when it is multiplied by the corresponding DP [32].

Essentially, a module is an object that contains the data and the methods that allow to implement the functional requirement FR. Estimating the information content with Function Points (FP) provides an advantage for composing the best solution; in fact, a numeric value, which can be compared to other possible solutions, can be assigned to elementary interoperable elements or bricks.

Through this technique, elementary functions and data structures are identified, containing all the elements needed to develop a customized system. In this case, Axiomatic Design combined with OOP and FP selects the most appropriate RPM solution for the specific goal.

This standardization mechanism, together with the subsequent bricks construction, make it easy to keep upto-date systems that will be able to follow technological evolution, continuous evolution of IT services and customer needs.

The development process of RPM solutions follows a flow, shown in Figure 3.

Starting from user clustering, a series of specific requests for each identified group is obtained. Through the QFD tool, such requests are translated into technical requirements by RPM developers in close connection with healthcare experts. The use of QFD also allows to understand the importance of technical features developed in customer optics [23-28].

Now, the functional requirements are identified and interoperable elements necessary for the development of RPM solutions (DPs) and their design constraints should be identified too.

Moreover, it is necessary to develop any needed interoperable elements if they are not available. Once all the needed elements are available, the system can be identified and optimized using AD axioms. In this case, the verification of the axiom of information is made by measuring the information content of system using the Function Point [2-3] technique. The assembly and the development of the selected best RPM solution can now be performed.

The presented framework might be a useful approach to ensure that development of new solutions shall already be partially guaranteed and will accompany the evolution of medicine and people's habits. Therefore, the application of QFD-AD and OOP is here performed in the healthcare environment, where "bricks" are customized and then assembled. The process becomes leaner and more robust in a context where the solutions developed are poorly declined into CNs.

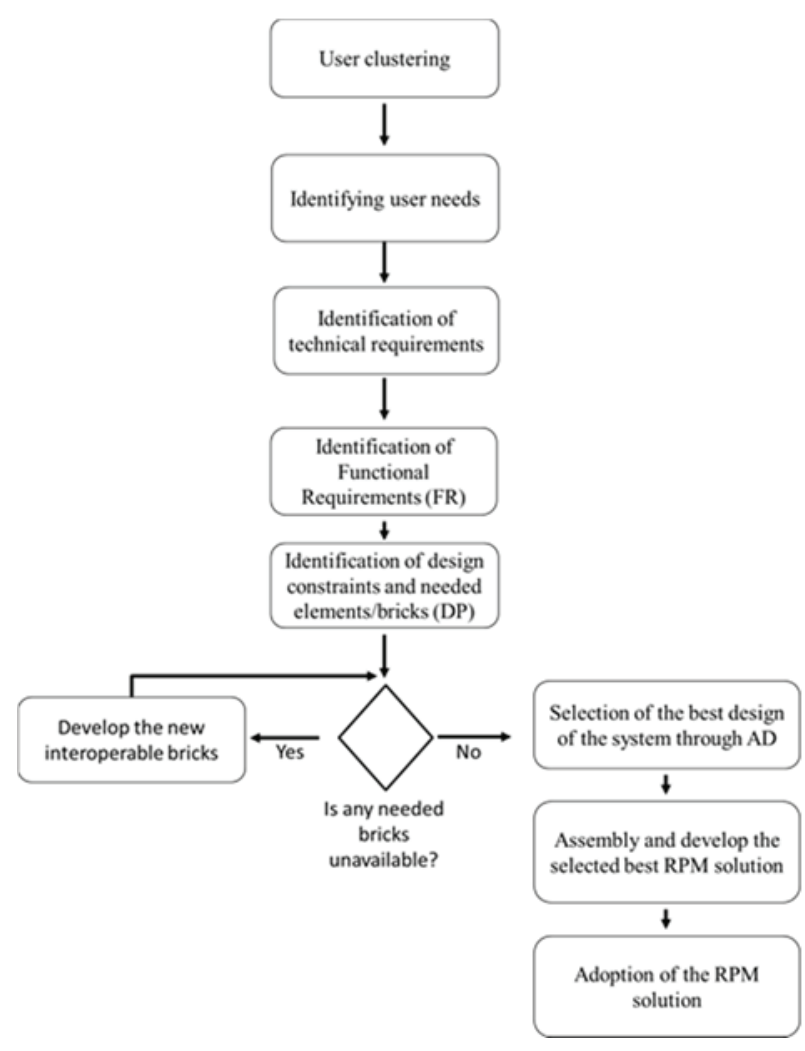

Fig. 3. Flow chart of the logical path.

\section{Conclusions}

Socio-economic convergence and development of reliable remote communications technology and devices make the prospect of massive introduction of RPM systems in healthcare sector very interesting. Despite the interest of many actors involved, most of the users' requests remain undone so far, as there is no system that cares about characterization of proposed solutions. In fact, most of the systems do not completely satisfy needs of either healthcare providers or users. The significant limit is certainly a lack of interoperable and standardized elements that allows a horizontal integration between different medical/specialized instruments and utilities aimed at improving quality of life. The proposed approach allows to overcome the aforementioned difficulties: it provides a method for selecting optimal RPM solutions, both for user satisfaction and simplification of the design process for the development of solutions, thanks to AD methodology. In fact, the presented process is useful to develop the minimal amount of software functionality and avoiding unnecessary implementations. Moreover, through the deployment of OOP and FP techniques, it is possible to realize an accurate planning of resources and costs, reducing the development time of RPM solutions. The holistic framework can be quickly refreshed, facilitating the exchange of technical information as well, thus following specialist medical and technological evolution.

In future works, this framework could be implemented, developed and tested, reaching full 
automation by providing software solutions capable of a mechanical selection of optimal bricks composition or a modification of the existing elementary bricks.

For this reason, the proposed model could be a useful approach to ensure that development of new solutions shall already be partially guaranteed and will accompany the evolution of medicine and people's habits.

\section{References}

1. A.A. Atienza., B.W. Hesse, D.H. Gustafson, R.T. Croyle, E-health research and patient-centered care examining theory, methods, and application, American Journal of Preventive Medicine 38, 1, 85-88 (2010)

2. A.J. Albrecht, Measuring Application Development Productivity, Proc. of the Joint SHARE/GUIDE/IBM, 83-92, (1979)

3. D. Garmus, IFPUG Function Point Counting Practices Manual, Release 4.1, (1999)

4. R. Meli, L. Santillo, Function point estimation methods: A comparative overview, Proc of FESMA, 99, 271-286, (1997)

5. F. Rolli, A. Giorgetti, P. Citti, Integration of Holistic Non-Conformities Management and Axiomatic Design: a case study in Italian Income Tax Returns Management, Proc CIRP 34, 256-262, (2015)

6. F. Rolli, A. Giorgetti, P. Citti, M. Rinaldi, Improvement of the compilation process of the Italian income certifications: a methodology based on the evaluation of the information content (Part 1), Proc CIRP 53, 5662, (2016)

7. F. Rolli, A. Giorgetti, P. Citti, M. Rinaldi, Improvement of the compilation process of the Italian income certifications: an application on the tax model of the year 2016 (Part 2), Proc CIRP 53, 63-69, (2016)

8. G. Arcidiacono, D. T. Matt, E. Rauch, Axiomatic Design of a Framework for the Comprehensive Optimization of Patient Flows in Hospitals, Journal of Healthcare Engineering 2017 (2017)

9. M. Kay, J. Santos, and M. Takane, mHealth: New horizons for health through mobile technologies, World Health Organization, vol. 64, 7, 66-71 (2011).

10. mHealth and Home Monitoring, Berg Insight (2014).

11. Connecting patient with providers A Pan-Canadian Study on RPM, (2014)

12. Canada Health Infoway Benefits Evaluation Indicators Technical Report, Version 2.0 (2012)

13. J. Antonio, M.A. Zamora-Izquierdo, A.F. Skarmeta, Interconnection framework for $m$ Health and remote monitoring based on the internet of things, IEEE Journal on Selected Areas in Communications 31, 9, 47-65, (2013)

14. M. Clarke, D. Bogia, K. Hassing, L. Steubesand, T. Chan, D. Ayyagari, Developing a standard for personal health devices based on 11073. EMBS 2007, 6174-6176, (2007)
15. M. Sandhya, R. M. Madhumitha, S. Sankar, Analysis of Threats in Interoperability of Medical Devices, International Journal of Medical, Health, Biomedical, Bioengineering and Pharmaceutical Engineering 11, 5, 264-267, (2017)

16. B. Pacifici, C. Parretti, G. Arcidiacono, A. Giorgetti, A. Girgenti, Conceptual framework for user based $R P M$, Proc. International Conference on Industrial Engineering and Operations Management, (2017)

17. G. Arcidiacono, J. Wang, K. Yang, Operating room adjusted utilization study, International Journal of Lean Six Sigma 6, 2, 111-137 (2015)

18. L. De Vito, O. Postolache, S. Rapuano, Measurements and Sensors for Motion Tracking, IEEE Instrumentation \& Measurement Magazine 17, 3, 30-38, (2014)

19. N.P. Suh, Axiomatic design - advancements and applications (Oxford University Press, 2001)

20. P.J. Clapis, J.D. Hinterstiener, Enhancing objectoriented software development through axiomatic design, Proc of ICAD (2000)

21. D.S. Cochran, J.T. Foley, B. Zhuming, Use of the manufacturing system design decomposition for comparative analysis and effective design of production systems, International Journal of Production Research 55, 3, 870-890, (2017)

22. G. Arcidiacono, N. Costantino, K. Yang, The AMSE Lean Six Sigma Governance Model, International Journal of Lean Six Sigma 7, 3, 233-266, (2016)

23. C. Cavallini,A. Giorgetti, P. Citti, F. Nicolaie, Integral aided method for material selection based on quality function deployment and comprehensive VIKOR algorithm, Materials and Design 47, 27-34, (2013)

24. A. Giorgetti, C. Cavallini, A. Ciappi, G. Arcidiacono, P. Citti, A holistic model for the proactive reduction of non-conformities within new industrial technologies, International Journal of Mechanical Engineering and Robotics Research, 6, 4, 313-317, (2017).

25. A. Giorgetti, C. Cavallini, G. Arcidiacono, P. Citti, $A$ mixed C-Vikor fuzzy approach for material selection during design phase: A case study in valve seats for high performance engine, International Journal of Applied Engineering Research 12, 12, 3117-3129, (2017).

26. A. Giorgetti, C. Cavallini, P. Citti, M, Delogu, A Novel Approach for Axiomatic-Based Design for the Environment, Axiomatic Design in Large Systems, 131-148 (2016)

27. A.M. Gonçalves Coelho, A.J. Mourão. Z.L. Pereira, Improving the use of QFD with Axiomatic Design, Concurrent Engineering 13, 3, pp. 233-239, (2005)

28. A. Girgenti, B, Pacifici, A.Ciappi, A. Giorgetti, An Axiomatic Design Approach for Customer Satisfaction through a Lean Start-up Framework, Proc. CIRP 53, 151-157, (2016)

29. G. Arcidiacono, A. Giorgetti, M. Pugliese, Axiomatic Design to improve PRM airport assistance, Proceedings, Proc CIRP 34, 106-111 (2015) 
30. G. Arcidiacono, P. Placidoli, Reality and illusion in Virtual Studios: Axiomatic Design applied to television recording, Proc CIRP 34, 137-142 (2015)
31. K. Dansky, D. Thompson, T. Sanner, A framework for evaluating eHealth research. Evaluation and Program Planning 29, 4, 397-404, (2006)

32. B.S EL-Haik, A. Shaout, Software Design for Six Sigma: A Roadmap for Excellence. (Wiley, 2010) 